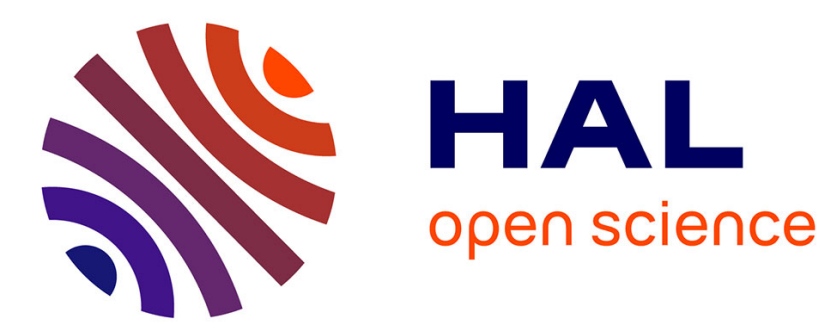

\title{
LMI-based output feedback control of singularly perturbed systems with guaranteed cost
}

Eduardo S Tognetti, Taís R Calliero, Irinel-Constantin Morarescu, Jamal

Daafouz

\section{- To cite this version:}

Eduardo S Tognetti, Taís R Calliero, Irinel-Constantin Morarescu, Jamal Daafouz. LMI-based output feedback control of singularly perturbed systems with guaranteed cost. 59th IEEE Conference on Decision and Control, CDC 2020, Dec 2020, Jeju Island, South Korea. hal-02956238

\section{HAL Id: hal-02956238 \\ https://hal.science/hal-02956238}

Submitted on 2 Oct 2020

HAL is a multi-disciplinary open access archive for the deposit and dissemination of scientific research documents, whether they are published or not. The documents may come from teaching and research institutions in France or abroad, or from public or private research centers.
L'archive ouverte pluridisciplinaire HAL, est destinée au dépôt et à la diffusion de documents scientifiques de niveau recherche, publiés ou non, émanant des établissements d'enseignement et de recherche français ou étrangers, des laboratoires publics ou privés. 


\title{
LMI-based output feedback control of singularly perturbed systems with guaranteed cost
}

\author{
Eduardo S. Tognetti, Taís R. Calliero, Irinel-Constantin Morărescu and Jamal Daafouz
}

\begin{abstract}
This paper addresses the problem of dynamic output feedback controller design for linear two time-scales dynamics. Unlike most of the works in the literature on singularly perturbed systems, we also consider the case where the state matrix of the fast dynamics is singular (nonstandard systems) and unstable. Our approach relies on decoupling the slow and fast dynamics of the closed-loop system and applying algebraic manipulations based on Finsler's Lemma to obtain $\varepsilon$ dependent and $\varepsilon$-independent controllers. The design conditions are computationally oriented since they are expressed in term of Linear Matrix Inequalities (LMIs). On top of this, a quadratic cost is guaranteed to be upper-bounded for all positive values of the singular parameter. The proposed conditions circumvent some drawbacks of the existing works on this topic by providing a dynamic controller that does not depend on the singular parameter. A numerical example illustrates the effectiveness of the proposed approach.
\end{abstract}

\section{INTRODUCTION}

Dynamical systems evolving on multiple time-scales are present in many fields of application and it is well known that, in this context, standard control techniques may lead to ill-conditioned strategies. To overcome the inherent numerical problems, singular perturbation framework [1] performs a time-scale decomposition in two smaller-order subsystems associated with the original dynamic. Based on this decoupling into slow and fast dynamics, many works focused on the state feedback control problem [1]. It is also worth mentioning the work [2] that deals with the problem of state feedback quadratic optimal control design for linear singularly perturbed systems.

The output feedback design, without the use of the separation principle (independent design of observer and controller gains), for singularly perturbed systems still remains a less explored field. One main reason is the difficulty to combine the control gains designed for the low-order subsystems to obtain a composite control for the global system. Some alternative solutions have been proposed. The work [3] applies a frequency-domain description of the two-time scale system to design an output controller. In [4], the authors present a set of $\varepsilon$-independent Riccati equations to design a two-time scale dynamic output feedback controller. The work [5] proposes high-gain observer-based controllers to achieve

This work was supported by the Brazilian agency FAPDF.

Eduardo S. Tognetti is with the Department of Electrical Engineering, University of Brasilia - UnB, 70910-900, Brasília, DF, Brazil, estognetti@ene.unb.br. Taís R. Calliero is with Faculty of Technology Gama, University of Brasilia - UnB, 70910-900, Brasília, DF, Brazil, tais.calliero@gmail.com. I.-C. Morărescu and J. Daafouz are with Université de Lorraine, CNRS, CRAN, F-54000 Nancy, France, constantin.morarescu@univ-lorraine.fr, jamal.daafouzeuniv-lorraine.fr robust output feedback controllers for a specific classes of nonlinear systems where the output does not depend on the system's fast state. More recently, [6] presented finitefrequency methods to design gains for the fast and slow subsystems however the composite controller is only valid for the original system if the feedthrough matrix of the controller has small magnitude.

We can cite as drawbacks of the above approaches, the inability to deal with nonstandard singularly perturbed systems, the lack of convex design conditions and the difficulty to impose dynamic controllers that do not dependent on the singular parameter. This last issue can be particularly important for the case where this parameter is not known (only an upper bound is available) or the actuators cannot respond to the fast variables resulting in controllers that are not implementable.

In this work we consider the problem of designing full and reduced-order dynamic output feedback controllers for singular perturbed linear systems with guaranteed cost. Differently from the current works in the literature and as an important feature, we propose a time-scale decomposition of the closed-loop system avoiding the design of a composite control law composed by the slow and fast components, separately designed. This approach allows to handle nonstandard singularly perturbed systems. As a novelty, we also propose the design of low order controllers, independent of the singular parameter, providing flexibility in the implementation.

Notation: The notation $\mathbb{R}^{n}, \mathbb{R}_{+}$and $\mathbb{R}^{n \times m}$ respectively denote the sets of $n$-dimensional real vectors, positive scalars, and $n \times m$-dimensional real matrices. For a matrix $A, A^{T}$ denotes the transpose of $A$ and, if $A$ is square, consider: $\operatorname{Tr}(A)$ stands for the trace of $A ; A^{-1}$ and $A^{-T}$ denote the inverse of $A$ and $A^{T}$, respectively; and $H e\{A\}=A+A^{T}$. The blockdiagonal matrix is denoted by $\operatorname{diag}(\cdot)$. The identity matrix of order $n$ is denoted by $I_{n}$ and the null $m \times n$ matrix is denoted by $0_{m, n}$ (or simply $I$ and 0 if no confusion arises). The symbol $\star$ denotes symmetric blocks in partitioned matrices.

\section{Preliminaries}

Consider the following singularly perturbed linear system:

$$
\begin{aligned}
{\left[\begin{array}{c}
\dot{x}(t) \\
\varepsilon \dot{z}(t)
\end{array}\right] } & =\left[\begin{array}{ll}
A_{11} & A_{12} \\
A_{21} & A_{22}
\end{array}\right]\left[\begin{array}{l}
x(t) \\
z(t)
\end{array}\right]+\left[\begin{array}{l}
B_{1} \\
B_{2}
\end{array}\right] u(t) \\
y(t) & =\left[\begin{array}{ll}
C_{1} & C_{2}
\end{array}\right]\left[\begin{array}{l}
x(t) \\
z(t)
\end{array}\right]
\end{aligned}
$$

where $x(t) \in \mathbb{R}^{n_{x}}$ and $z(t) \in \mathbb{R}^{n_{z}}$ are the states, $u(t) \in \mathbb{R}^{n_{u}}$ is the control input, $y(t) \in \mathbb{R}^{n_{y}}$ is the measured output and $\varepsilon>0$ is a singular perturbation parameter. 
Define the following quadratic cost function associated with the dynamic (1):

$$
J=\int_{0}^{\infty} x(t)^{T} Q_{x} x(t)+z(t)^{T} Q_{z} z(t)+u(t)^{T} R u(t) d t
$$

where $Q_{x} \geq 0, Q_{z} \geq 0$ and $R>0$ are symmetric matrices that weights the effort of the control action and convergence of the trajectories. The objective is to design a dynamic output feedback (DOF) controller that minimizes an upper bound to the quadratic cost (2) for any sufficiently small parameter $\varepsilon$, that is, there exists $\varepsilon^{*}>0$ such that the guaranteed cost $\bar{J}$ satisfies

$$
\bar{J} \geq J, \quad \forall \varepsilon \in\left(0, \varepsilon^{*}\right)
$$

Observe that the cost (2) can be rewritten as

$$
J=\int_{0}^{\infty} y_{z}(t)^{T} y_{z}(t) d t
$$

with

$$
y_{z}(t)=\left[\begin{array}{ll}
C_{z 1} & C_{z 2}
\end{array}\right]\left[\begin{array}{l}
x(t) \\
z(t)
\end{array}\right]+D u(t)
$$

and

$$
\left[\begin{array}{c|c}
C_{z 1} & C_{z 2}
\end{array}\right]=\left[\begin{array}{c|c}
\sqrt{Q_{x}} & 0 \\
0 & \sqrt{Q_{z}} \\
0 & 0
\end{array}\right], \quad D=\left[\begin{array}{c}
0 \\
0 \\
\sqrt{R}
\end{array}\right] .
$$

We consider in this work two classes of controllers: $\varepsilon$-dependent and $\varepsilon$-independent DOF controllers. The $\varepsilon$ dependent full-order DOF controller has the following structure:

$$
\begin{aligned}
{\left[\begin{array}{c}
\dot{x}_{c}(t) \\
\varepsilon \dot{z}_{c}(t)
\end{array}\right] } & =\left[\begin{array}{ll}
A_{c 11} & A_{c 12} \\
A_{c 21} & A_{c 22}
\end{array}\right]\left[\begin{array}{l}
x_{c}(t) \\
z_{c}(t)
\end{array}\right]+\left[\begin{array}{l}
B_{c 1} \\
B_{c 2}
\end{array}\right] y(t) \\
u(t) & =\left[\begin{array}{ll}
C_{c 1} & C_{c 2}
\end{array}\right]\left[\begin{array}{l}
x_{c}(t) \\
z_{c}(t)
\end{array}\right]+D_{c} y(t)
\end{aligned}
$$

where $x_{c}(t) \in \mathbb{R}^{n_{x}}$ and $z_{c}(t) \in \mathbb{R}^{n_{z}}$ are the states of the controller. Note that the controller has a two time-scale property and we suppose that the actuator of a plant modeled by (1) is able to respond to the fast variable $z_{c}(t)$ presented in the control signal $u(t)$.

The closed-loop system formed by (1) and (5) is given by

$$
\begin{aligned}
{\left[\begin{array}{c}
\dot{\xi}(t) \\
\varepsilon \dot{\mu}(t)
\end{array}\right] } & =\left[\begin{array}{cc}
\tilde{A}_{11} & \tilde{A}_{12} \\
\tilde{A}_{21} & \tilde{A}_{22}
\end{array}\right]\left[\begin{array}{l}
\xi(t) \\
\mu(t)
\end{array}\right] \\
y_{z}(t) & =\left[\begin{array}{ll}
\tilde{C}_{z 1} & \tilde{C}_{z 2}
\end{array}\right]\left[\begin{array}{l}
\xi(t) \\
\mu(t)
\end{array}\right],
\end{aligned}
$$

with $\xi(t)=\left(x(t)^{T}, x_{c}(t)^{T}\right)^{T} \in \mathbb{R}^{2 n_{x}}, \mu(t)=\left(z(t)^{T}, z_{c}(t)^{T}\right)^{T} \in$ $\mathbb{R}^{2 n_{z}}$, and matrices given by

$\tilde{A}_{i j}=\left[\begin{array}{cc}A_{i j}+B_{i} D_{c} C_{j} & B_{i} C_{c j} \\ B_{c i} C_{j} & A_{c i j}\end{array}\right], \quad \tilde{C}_{z i}=\left[\begin{array}{ll}C_{z i}+D D_{c} C_{i} & D C_{c i}\end{array}\right]$,

$i, j=1,2$.

Although the $\varepsilon$-dependent output feedback controller performs very well, it may be difficult to implement due to fast actuation requirement. Motivated by this observation, we will also design $\varepsilon$-independent output feedback controllers that provides slow actuation. In this part we suppose that $\varepsilon$ is unknown but upper bounded by a known value.

The following $\varepsilon$-independent reduced-order DOF controller is considered:

$$
\begin{aligned}
\dot{x}_{c}(t) & =A_{c} x_{c}(t)+B_{c} y(t) \\
u(t) & =C_{c} x_{c}(t)+D_{c} y(t),
\end{aligned}
$$

where $x_{c}(t) \in \mathbb{R}^{n_{x}}$.

The closed-loop system composed by (1) and the controller (8) is given by (6) with $\xi(t)=\left(x(t)^{T}, x_{c}(t)^{T}\right)^{T}, \mu(t)=$ $z(t)$, and matrices:

$$
\begin{array}{cc}
\tilde{A}_{11}=\left[\begin{array}{cc}
A_{11}+B_{1} D_{c} C_{1} & B_{1} C_{c} \\
B_{c} C_{1} & A_{c}
\end{array}\right], & \tilde{A}_{12}=\left[\begin{array}{c}
A_{12}+B_{1} D_{c} C_{2} \\
B_{c} C_{2}
\end{array}\right] \\
\tilde{A}_{21}=\left[\begin{array}{ll}
A_{21}+B_{2} D_{c} C_{1} & B_{2} C_{c}
\end{array}\right], & \tilde{A}_{22}=A_{22}+B_{2} D_{c} C_{2}, \\
\tilde{C}_{z 1}=\left[\begin{array}{ll}
C_{z 1}+D D_{c} C_{1} & D C_{c}
\end{array}\right], & \tilde{C}_{z 2}=C_{z 2}+D D_{c} C_{2} .
\end{array}
$$

We are now ready to state the problem addressed in this paper.

Problem 1 For the singularly perturbed linear system (1), find a DOF controller (5) (respectively, (8)) such that the closed-loop system (6) is asymptotically stable and minimizes the guaranteed cost $\bar{J}$ such that (3) holds.

The following lemmas will be useful for the further developments.

Lemma 1 Let a symmetric matrix $M_{0} \in \mathbb{R}^{n \times n}$ and matrices $M_{1} \in \mathbb{R}^{m \times n}$ and $M_{2} \in \mathbb{R}^{m \times n}$. Then the following conditions are equivalent:

$$
\begin{aligned}
& \text { i. } M_{0}+\operatorname{He}\left\{M_{1}^{T} M_{2}\right\}<0 \\
& \text { ii. } \exists N_{1} \in \mathbb{R}^{n \times m} \text { and } N_{2} \in \mathbb{R}^{m \times m}: \\
& {\left[\begin{array}{cc}
M_{0} & M_{2}^{T} \\
M_{2} & 0
\end{array}\right]+\operatorname{He}\left\{\left[\begin{array}{l}
N_{1} \\
N_{2}
\end{array}\right]\left[\begin{array}{ll}
M_{1} & -I
\end{array}\right]\right\}<0 .}
\end{aligned}
$$

Proof: The equivalence can be demonstrated by the Finsler's Lemma [7] and is omitted for the sake of brevity and due to space limitations.

Lemma 2 ( [8]) Consider two symmetric matrices with the following structure

$$
\Theta=\left[\begin{array}{cc}
\Theta_{11} & \Theta_{12} \\
\star & \Theta_{22}
\end{array}\right], \quad \Upsilon=\left[\begin{array}{cc}
0 & \Upsilon_{12} \\
\star & \Upsilon_{22}
\end{array}\right]
$$

with $\Upsilon_{22}$ nonsingular. The following conditions are equivalent.

1) There exists $\varepsilon^{*}$ such that

$$
\Theta+\varepsilon^{-1} \Upsilon<0
$$

holds for all $\varepsilon \in\left(0, \varepsilon^{*}\right)$.

2) The conditions

$$
\Upsilon_{12}=0, \quad \Theta_{11}<0, \quad \Upsilon_{22}<0
$$

simultaneously hold. 


\section{MAIN RESULTS}

In the sequel we use the Lyapunov function

$$
V(\xi, \mu)=\left[\begin{array}{l}
\xi \\
\mu
\end{array}\right]^{T} W^{-1}\left[\begin{array}{l}
\xi \\
\mu
\end{array}\right],
$$

with $W \in \mathbb{R}^{2 n_{x}+2 n_{z}}$ a symmetric positive definite matrix, $\xi(t)=\left(x(t)^{T}, x_{c}(t)^{T}\right)^{T}$ and $\mu(t)=\left(z(t)^{T}, z_{c}(t)^{T}\right)^{T}$. If there exist a matrix $W=W^{T}>0$ and a scalar $\gamma>0$ such that the following inequality holds

$$
\dot{V}(\xi(t), \mu(t))+\gamma^{-1} y_{z}(t)^{T} y_{z}(t) \leq 0,
$$

then the integration of (13) over that interval $[0, \infty)$ implies

$$
J \leq \gamma V(\xi(0), \mu(0)),
$$

that is, the cost $J$ is upper limited by the initial condition $(\xi(0), \mu(0))$ weighted by $\gamma W^{-1}$. For the minimization of the guaranteed cost $\bar{J}$ such that (3) holds we can exploit the fact the initial condition of the controller can be set arbitrarily to zero and minimize $\gamma$ and the trace of $W^{-1}$.

Usually, in the literature of singularly perturbed systems, the control design is performed for each subsystem (slow and fast lower-order dynamics) and their combination allows obtaining a composite control for the global system [1]. This approach requires $A_{22}$ to be nonsingular and works very well for the state feedback control problem [1]. For the DOF control problem, this is also the approach followed in [4] using a Riccati formulation, but the LMI-based design becomes intricate. In this work we adopt a different approach, we propose a time-scale decomposition of the closed-loop system to define two $\varepsilon$-independent subsystems associated with (6). As a consequence, we can deal with nonstandard singularly perturbed systems where matrix $A_{22}$ is not required to be non-singular.

Our objective in what follows is to decouple the slow and fast dynamics that are combined in the overall system. Let us recall that the reduced-order(slow) system is obtained in [1] by setting $\varepsilon=0$ in (6) and expressing the slow part of $\mu(t)$, denoted by $\mu_{s}(t)$, in terms of the slow part of $\xi(t)$, denoted by $\xi_{s}(t)$. If $\tilde{A}_{22}$ is non-singular, we can write

$$
\mu_{s}(t)=-G \xi_{s}(t),
$$

where $G=\tilde{A}_{22}^{-1} \tilde{A}_{21}$, and, therefore, the reduced-order (slow) system is

$$
\dot{\xi}_{s}(t)=A_{s} \xi_{s}(t), \quad \xi_{s}(0)=\xi(0),
$$

where $A_{S}=\tilde{A}_{11}-\tilde{A}_{12} G$.

On the other hand, the boundary-layer (fast) system is defined by treating $\xi(t)$ as a constant variable and removing the slow bias from $\mu(t)$, that is, $\mu_{f}(t)=\mu(t)-\mu_{s}(t)$. This leads at

$$
\varepsilon \dot{\mu}_{f}(t)=\tilde{A}_{22} \mu_{f}(t), \quad \mu_{f}(0)=\mu(0)+G \xi(0) .
$$

Consequently,

$$
\begin{aligned}
y_{z}(t) & =\tilde{C}_{z 1} \xi_{s}(t)+\tilde{C}_{z 2}\left(\mu_{f}(t)-G \xi_{s}(t)\right) \\
& =C_{s} \xi_{s}(t)+\tilde{C}_{z 2} \mu_{f}(t)
\end{aligned}
$$

where $C_{s}=\tilde{C}_{z 1}-\tilde{C}_{z 2} G$.

System (15) is well-defined only if $\tilde{A}_{22}$ is nonsingular. To impose this condition we design $\left(A_{c 22}, B_{c 2}, C_{c 2}, D_{c}\right)$ such that $\tilde{A}_{22}$ is Hurwitz, that is, the fast system (16) is asymptotically stable.

Next, we present $\varepsilon$-independent conditions for asymptotically stability with guaranteed cost of the close-loop system (6) in terms of its slow and fast decomposition.

Lemma 3 Suppose there exist symmetric positive definite matrices $W_{1} \in \mathbb{R}^{n_{x} \times n_{x}}$ and $W_{2} \in \mathbb{R}^{n_{z} \times n_{z}}$, and a scalar $\gamma \in \mathbb{R}_{+}$ verifying the following conditions:

$$
\begin{gathered}
{\left[\begin{array}{cc}
A_{s} W_{1}+W_{1} A_{s}^{T} & \star \\
C_{s} W_{1} & -\gamma I
\end{array}\right]<0,} \\
\tilde{A}_{22} W_{2}+W_{2} \tilde{A}_{22}^{T}<0 .
\end{gathered}
$$

Then, there exists $\varepsilon^{*}>0$ such that for all $\varepsilon \in\left(0, \varepsilon^{*}\right)$ the closed-loop system (6) is asymptotically stable with guaranteed cost given by $\bar{J}=\gamma \xi(0)^{T} W_{1}^{-1} \xi(0)+\gamma \mu_{f}(0)^{T} W_{2}^{-1} \mu_{f}(0)$.

Proof: It follows from the proof of Theorem 1 of [8] by considering the system (6) rewritten as

$$
\begin{aligned}
{\left[\begin{array}{c}
\dot{\xi} \\
\dot{\mu}
\end{array}\right] } & =\tilde{A}(\varepsilon)\left[\begin{array}{l}
\xi \\
\mu
\end{array}\right], \quad \tilde{A}(\varepsilon)=\left[\begin{array}{cc}
\tilde{A}_{11} & \tilde{A}_{12} \\
0 & 0
\end{array}\right]+\varepsilon^{-1}\left[\begin{array}{cc}
0 & 0 \\
\tilde{A}_{21} & \tilde{A}_{22}
\end{array}\right], \\
y_{z} & =\tilde{C}_{z}\left[\begin{array}{c}
\xi \\
\mu
\end{array}\right], \quad \tilde{C}_{z}=\left[\begin{array}{ll}
\tilde{C}_{z 1} & \tilde{C}_{z 2}
\end{array}\right] .
\end{aligned}
$$

Condition (13) with the Lyapunov function given by (12) is equivalent to

$$
W \tilde{A}(\varepsilon)^{T}+\tilde{A}(\varepsilon) W+\gamma^{-1} W \tilde{C}_{z}^{T} \tilde{C}_{z} W<0 .
$$

Consider $W$ with the following partition

$$
W=\left[\begin{array}{cc}
W_{1} & -W_{1} G^{T} \\
\star & W_{2}+G W_{1} G^{T}
\end{array}\right] .
$$

If we replace (21) in (20), we obtain an expression in the form of (10) where $\Upsilon_{22}=\tilde{A}_{22} W_{2}+W_{2} \tilde{A}_{22}^{T}$ and $\Theta_{11}=A_{s} W_{1}+$ $W_{1} A_{s}^{T}+\gamma^{-1} W_{1} C_{s}^{T} C_{s} W_{1}$. Then, by Lemma 2 and applying the Schur complement in $\Theta_{11}<0$, conditions (18)-(19) are equivalent to (11). Observe that $W$ is a candidate Lyapunov matrix in (20) since $W>0$ is assured by $W_{1}>0$ and, by Schur complement, $W_{2}>0$.

From (21), one has

$$
W^{-1}=\left[\begin{array}{cc}
W_{1}^{-1}+G^{T} W_{2}^{-1} G & G^{T} W_{2}^{-1} \\
\star & W_{2}^{-1}
\end{array}\right]
$$

then, considering (14), one has

$$
\begin{aligned}
J & \leq \gamma\left[\begin{array}{l}
\xi(0) \\
\mu(0)
\end{array}\right]^{T}\left[\begin{array}{cc}
W_{1}^{-1}+G^{T} W_{2}^{-1} G & G^{T} W_{2}^{-1} \\
\star & W_{2}^{-1}
\end{array}\right]\left[\begin{array}{l}
\xi(0) \\
\mu(0)
\end{array}\right] \\
& =\gamma \xi(0)^{T} W_{1}^{-1} \xi(0)+\gamma(\mu(0)+G \xi(0))^{T} W_{2}^{-1}(\mu(0)+G \xi(0)) \\
& =\gamma \xi(0)^{T} W_{1}^{-1} \xi(0)+\gamma \mu_{f}(0)^{T} W_{2}^{-1} \mu_{f}(0)
\end{aligned}
$$

Observe from Lemma 3 that $V_{1}\left(\xi_{s}\right)=\xi_{s}^{T} W_{1}^{-1} \xi_{s}$ and $V_{2}\left(\mu_{f}\right)=\mu_{f}^{T} W_{2}^{-1} \mu_{f}$ can be seen as Lyapunov functions for stability analysis of the slow and fast systems, respectively, 
for all $\varepsilon \in\left(0, \varepsilon^{*}\right)$. This fully agrees with the arguments of time-decomposition provided in [1]. However, conditions of Lemma 3 are non-convex for the design of the controller (5).

Observe that the inverse of $\tilde{A}_{22}$ in the conditions (18) and (19) impose a difficulty. One possible solution is to design the gains $\left(A_{c 22}, B_{c 2}, C_{c 2}, D_{c}\right)$ such that (19) holds and then solve (18). However, the design in two independent steps is not convenient since the gains obtained to stabilize the fast systems may not be suitable to stabilize the slow one or may yield a conservative solution for the guaranteed cost $J$. Therefore, we propose an LMI-based one-step procedure for Lemma 3. We will first detail the design of $\varepsilon$-dependent DOF controllers and then consider $\varepsilon$-independent ones.

\section{A. $\varepsilon$-dependent DOF controllers}

Consider the following parametrization for the Lyapunov matrices adapted from [9]:

$$
W_{i}=\left[\begin{array}{cc}
X_{i} & -X_{i} \\
-X_{i} & H_{i}
\end{array}\right], \quad W_{i}^{-1}=\left[\begin{array}{cc}
Y_{i} & V_{i} \\
V_{i} & V_{i}
\end{array}\right], \quad i=1,2,
$$

where $X_{1} \in \mathbb{R}^{n_{x} \times n_{x}}, Y_{1} \in \mathbb{R}^{n_{x} \times n_{x}}, X_{2} \in \mathbb{R}^{n_{z} \times n_{z}}, Y_{2} \in \mathbb{R}^{n_{z} \times n_{z}}$ are symmetric positive definite matrices, $V_{i}=Y_{i}-X_{i}^{-1}$ and $H_{i}=X_{i}+V_{i}^{-1}$.

We observe that matrices $\tilde{A}_{11}, \tilde{A}_{12}, \tilde{A}_{21}$ and $\tilde{A}_{22}$ have the same structure, then the product with the Lyapunov matrices can be handled with the congruence transformation and the change of variables proposed by [9]. Define the following non-singular matrices

$$
T_{i}=\left[\begin{array}{cc}
I & Y_{i} \\
0 & V_{i}^{T}
\end{array}\right], \quad i=1,2 .
$$

Then, pre- and post-multiplying (18) by $\operatorname{diag}\left(T_{1}^{T}, I\right)$ and its transpose, respectively, and introducing the terms $T_{2} T_{2}^{-1}=$ $I$ and $W_{2} T_{2} T_{2}^{-1} W_{2}^{-1}=I$ in appropriate positions, one can observe that the inequality (18) is equivalent to

$$
\left[\begin{array}{cc}
\operatorname{He}\left\{T_{1}^{T} \tilde{A}_{11} W_{1} T_{1}-\Delta_{1}\right\} & \star \\
\tilde{C}_{z 1} W_{1} T_{1}-\Delta_{2} & -\gamma I
\end{array}\right]<0
$$

where

$$
\begin{aligned}
& \Delta_{1}=T_{1}^{T} \tilde{A}_{12}\left(W_{2} T_{2} T_{2}^{-1} W_{2}^{-1}\right) \tilde{A}_{22}^{-1}\left(T_{2}^{-T} T_{2}^{T}\right) \tilde{A}_{21} W_{1} T_{1} \\
& \Delta_{2}=\tilde{C}_{z 2}\left(W_{2} T_{2} T_{2}^{-1} W_{2}^{-1}\right) \tilde{A}_{22}^{-1}\left(T_{2}^{-T} T_{2}^{T}\right) \tilde{A}_{21} W_{1} T_{1},
\end{aligned}
$$

and matrices $\tilde{A}_{i j}$ and $\tilde{C}_{z i}$ are given by (7). Inequality (24) can be rewritten as

$$
\left[\begin{array}{cc}
\operatorname{He}\left\{\Psi_{11}-\Psi_{12} \Psi_{22}^{-1} \Psi_{21}\right\} & \star \\
\Lambda_{1}-\Lambda_{2} \Psi_{22}^{-1} \Psi_{21} & -\gamma I
\end{array}\right]<0,
$$

where $\Psi_{i j}=T_{i}^{T} \tilde{A}_{i j} W_{j} T_{j}$ and $\Lambda_{i}=\tilde{C}_{z i} W_{i} T_{i}, i, j=1,2$. If we define the following variables

$$
\begin{gathered}
L_{i}=\left(D_{c} C_{i}-C_{c i}\right) X_{i}, \quad F_{i}=Y_{i} B_{i} D_{c}+V_{i} B_{c i} \\
Q_{i j}=\left[\begin{array}{ll}
Y_{i} & V_{i}
\end{array}\right] \tilde{A}_{i j}\left[\begin{array}{c}
X_{j} \\
-X_{j}
\end{array}\right],
\end{gathered}
$$

the terms $\Psi_{i j}$ and $\Lambda_{i}$ can be rewritten as

$$
\begin{aligned}
\Psi_{i j} & =\left[\begin{array}{cc}
A_{i j} X_{j}+B_{i} L_{j} & A_{i j}+B_{i} D_{c} C_{j} \\
Q_{i j} & Y_{i} A_{i j}+F_{i} C_{j}
\end{array}\right], \\
\Lambda_{i} & =\left[\begin{array}{ll}
C_{z i} X_{i}+D L_{i} & C_{z i}+D D_{c} C_{i}
\end{array}\right] .
\end{aligned}
$$

One can see in (27) that $\Psi_{i j}$ and $\Lambda_{i}$ are affine in the variables $X_{i}, Y_{i}, L_{i}, F_{i}, Q_{i j}, i, j=1,2$, and $D_{c}$. Consequently, we can apply Lemma 1 to decouple the product involving the terms $\Psi_{i j}$ and $\Lambda_{i}$. Our first main result is given below and it basically presents sufficient conditions for Lemma 3 . to hold true.

Theorem 1 Suppose that there exist symmetric positive definite matrices $X_{1} \in \mathbb{R}^{n_{x} \times n_{x}}, Y_{1} \in \mathbb{R}^{n_{x} \times n_{x}}, X_{2} \in \mathbb{R}^{n_{z} \times n_{z}}, Y_{2} \in$ $\mathbb{R}^{n_{z} \times n_{z}}$, a scalar $\gamma \in \mathbb{R}_{+}$and matrices $F_{1} \in \mathbb{R}^{n_{x} \times n_{y}}, L_{1} \in$ $\mathbb{R}^{n_{u} \times n_{x}}, F_{2} \in \mathbb{R}^{n_{z} \times n_{y}}, L_{2} \in \mathbb{R}^{n_{u} \times n_{z}} Q_{11} \in \mathbb{R}^{n_{x} \times n_{x}}, Q_{12} \in$ $\mathbb{R}^{n_{x} \times n_{z}}, Q_{21} \in \mathbb{R}^{n_{z} \times n_{x}}, Q_{22} \in \mathbb{R}^{n_{z} \times n_{z}}$, and $D_{c} \in \mathbb{R}^{n_{u} \times n_{y}}$, a given scalar $\varsigma>0$, and a given matrix $\mathscr{I} \in \mathbb{R}^{2 n_{x} \times 2 n_{z}}$, such that

$$
\begin{gathered}
{\left[\begin{array}{cc}
X_{i} & I \\
I & Y_{i}
\end{array}\right]>0, \quad i=1,2} \\
\Xi<0
\end{gathered}
$$

where

$$
\begin{gathered}
\Xi=\left[\begin{array}{ccc}
\operatorname{He}\left\{\Psi_{11}+\Theta\right\} & \star & \star \\
\Omega & \varsigma \operatorname{He}\left\{\Psi_{22}\right\} & \star \\
\Lambda_{1}+\Lambda_{2} \mathscr{I}^{T} & \varsigma \Lambda_{2} & -\gamma I
\end{array}\right], \\
\Theta=\mathscr{I} \Psi_{12}^{T}, \quad \Omega=\Psi_{21}+\Psi_{22} \mathscr{I}^{T}+\varsigma \Psi_{12}^{T},
\end{gathered}
$$

$\Psi_{i j}$ and $\Lambda_{i}$ given in (27). Then, there exists $\varepsilon^{*}>0$ such that for all $\varepsilon \in\left(0, \varepsilon^{*}\right)$ the controller (5) with gains

$$
\begin{gathered}
{\left[\begin{array}{cc}
A_{c i j} & B_{c i} \\
C_{c j} & D_{c}
\end{array}\right]=\left[\begin{array}{cc}
V_{i}^{-1} & -V_{i}^{-1} Y_{i} B_{i} \\
0 & I
\end{array}\right]\left[\begin{array}{cc}
Q_{i j}-Y_{i} A_{i j} X_{j} & F_{i} \\
L_{j} & D_{c}
\end{array}\right]} \\
\times\left[\begin{array}{cc}
-X_{j}^{-1} & 0 \\
C_{j} & I
\end{array}\right], \quad V_{i}=Y_{i}-X_{i}^{-1}, \quad i, j=1,2,
\end{gathered}
$$

makes the closed-loop system (6) asymptotically stable with guaranteed cost $\bar{J}=\gamma \xi(0)^{T} W_{1}^{-1} \xi(0)+\gamma \mu_{f}(0)^{T} W_{2}^{-1} \mu_{f}(0)$.

Proof: First, note that inequality (28) is equivalent to $T_{i}^{T} W_{i} T_{i}>0$, for $W_{1}$ and $W_{2}$ as in (22) and $T_{1}$ and $T_{2}$ as in (23), then $W_{i}>0, i=1,2$, since $T_{i}$ is non-singular. Observe also that (28) implies, by the Schur complement, $V_{i}$ non-singular.

The inequality (25) can be written as condition i. of Lemma 1 with

$$
M_{0}=\left[\begin{array}{cc}
\operatorname{He}\left\{\Psi_{11}\right\} & \star \\
\Lambda_{1} & -\gamma I
\end{array}\right], \quad M_{1}^{T}=-\left[\begin{array}{c}
\Psi_{12} \\
\Lambda_{2}
\end{array}\right] \Psi_{22}^{-1},
$$

$M_{2}=\left[\begin{array}{ll}\Psi_{21} & 0\end{array}\right]$, and inequality (29) can be written as condition ii. of Lemma 1 with $N_{1}^{T}=-\Psi_{22}\left[\begin{array}{ll}\mathscr{I}^{T} & 0\end{array}\right]$ and $N_{2}=-\varsigma^{T} \Psi_{22}^{T}$. Therefore, by Lemma 1, if (29) holds, then (25) is satisfied. By considering the change of variables (22) and (26), rewriting (25) as (24), pre- and post-multiplying (24) by $\operatorname{diag}\left(T_{1}^{-T}, I\right)$ and its transpose, respectively, one obtains (18). Pre- and post-multiplying $\Xi$ by $\left[0 T_{2}^{-T} 0\right]$, then (29) implies (19) for any $\varsigma>0$.

Then, by Lemma 3, we conclude that there exists $\varepsilon^{*}$ such that the closed-loop system (6) is asymptotically stable with a guaranteed cost $\bar{J}$ given by $\gamma \xi(0)^{T} W_{1}^{-1} \xi(0)+$ $\gamma \mu_{f}(0)^{T} W_{2}^{-1} \mu_{f}(0)$ for all $\varepsilon \in\left(0, \varepsilon^{*}\right)$. 
Remark 1 Condition (29) becomes an LMI for fixed values of $\zeta$ and $\mathscr{I}$. Matrix $\mathscr{I}$ is a given matrix used to adjust the dimension in (29) for the case $n_{x} \neq n_{z}$. We remarked good numerical results for the choice $\mathscr{I}=0$ or $\mathscr{I}=\tau \mathbb{I}$, where $\mathbb{I} \in \mathbb{R}^{2 n_{x} \times 2 n_{z}}$ is the identity matrix for $n_{x}=n_{z}, \mathbb{I}=$ $\left[\begin{array}{ll}I & 0\end{array}\right]$ for $n_{x}<n_{z}$ and $\mathbb{I}=\left[\begin{array}{ll}I & 0\end{array}\right]^{T}$ for $n_{x}>n_{z}$, where 0 is a rectangular matrix with appropriate dimension. The scalar $\tau \in \mathbb{R}$ represents an extra degree of freedom.

Remark 2 The inequality (25) can be also written as condition i. of Lemma 1 with

$$
M_{1}=\Psi_{22}^{-1}\left[\begin{array}{ll}
\Psi_{21} & 0
\end{array}\right], \quad M_{2}^{T}=-\left[\begin{array}{c}
\Psi_{12} \\
\Lambda_{2}
\end{array}\right]
$$

and $M_{0}$ as in (33). Therefore, the choice $N_{1}^{T}=-\left[\begin{array}{ll}\mathscr{I} & 0\end{array}\right] \Psi_{22}^{T}$ and $N_{2}=-\varsigma^{\top} \Psi_{22}^{T}$ yields $\Xi$ in (30) with

$$
\Theta=\mathscr{I} \Psi_{21}, \quad \Omega=\Psi_{12}^{T}+\Psi_{22}^{T} \mathscr{I}^{T}+\varsigma \Psi_{21}
$$

Finally, we observe that conditions of Theorem 1 with (31) and (34) are not equivalent, thus one condition may be feasible and other not and different costs may be obtained for a given initial condition.

\section{B. $\varepsilon$-independent DOF controllers}

First, observe that inequality (18) with matrices (9) is equivalent to

$$
\left[\begin{array}{cc}
\operatorname{He}\left\{T_{1}^{T} \tilde{A}_{11} W_{1} T_{1}-T_{1}^{T} \tilde{A}_{12} \tilde{A}_{22}^{-1} \tilde{A}_{21} W_{1} T_{1}\right\} & \star \\
\tilde{C}_{z 1} W_{1} T_{1}-\tilde{C}_{z 2} \tilde{A}_{22}^{-1} \tilde{A}_{21} W_{1} T_{1} & -\gamma I
\end{array}\right]<0
$$

or, alternatively,

$$
\left[\begin{array}{cc}
\operatorname{He}\left\{\Psi_{11}-\varphi_{12} \tilde{A}_{22}^{-1} \varphi_{21}\right\} & \star \\
\Lambda_{1}-\left(C_{z 2}+D D_{c} C_{2}\right) \tilde{A}_{22}^{-1} \varphi_{21} & -\gamma I
\end{array}\right]<0
$$

with

$$
\begin{aligned}
\varphi_{12} & =\left[\begin{array}{ll}
\left(A_{12}+B_{1} D_{c} C_{2}\right)^{T} & A_{12}^{T} Y_{1}+C_{2}^{T} F_{1}^{T}
\end{array}\right], \\
\varphi_{21} & =\left[\begin{array}{ll}
A_{21} X_{1}+B_{2} L_{1} & A_{21}+B_{2} D_{c} C_{1}
\end{array}\right] .
\end{aligned}
$$

Following similar steps as above, the next theorem presents sufficient conditions for the design of (8).

Theorem 2 If there exist symmetric positive definite matrices $X_{1} \in \mathbb{R}^{n_{x} \times n_{x}}, Y_{1} \in \mathbb{R}^{n_{x} \times n_{x}}, W_{2} \in \mathbb{R}^{n_{z} \times n_{z}}$, a scalar $\gamma \in \mathbb{R}_{+}$ and matrices $F_{1} \in \mathbb{R}^{n_{x} \times n_{y}}, L_{1} \in \mathbb{R}^{n_{u} \times n_{x}}, Q_{11} \in \mathbb{R}^{n_{x} \times n_{x}}, D_{c} \in$ $\mathbb{R}^{n_{u} \times n_{y}}$, a given positive scalar $\varsigma$, and a given matrix $\mathscr{I} \in$ $\mathbb{R}^{2 n_{x} \times n_{z}}$, such that (28) and

$$
\begin{aligned}
& {\left[\begin{array}{ccc}
\operatorname{He}\left\{\Psi_{11}+\mathscr{I} \varphi_{12}\right\} & \star & \star \\
\varphi_{21}+\tilde{A}_{22} \mathscr{I}^{T}+\varsigma \varphi_{12} & \varsigma \mathrm{He}\left\{\tilde{A}_{22}\right\} & \star \\
\Lambda_{1}+\left(C_{z 2}+D D_{c} C_{2}\right) \mathscr{I}^{T} & \varsigma\left(C_{z 2}+D D_{c} C_{2}\right) & -\gamma I
\end{array}\right]<0,} \\
& {\left[\begin{array}{cc}
\mathrm{He}\left\{A_{22} W_{2}+B_{2} D_{c} C_{2}\right\} & \star \\
C_{2} W_{2}-C_{2}+D_{c}^{T} B_{2}^{T} & -2 I
\end{array}\right]<0}
\end{aligned}
$$

hold, then there exists $\varepsilon^{*}>0$ such that for all $\varepsilon \in\left(0, \varepsilon^{*}\right)$ the closed-loop system (6) is asymptotically stable with guaranteed cost $\bar{J}=\gamma \xi(0)^{T} W_{1}^{-1} \xi(0)+\gamma \mu_{f}(0)^{T} W_{2}^{-1} \mu_{f}(0)$ and controller gains of (8) given by (32) with $i=j=1$.
Proof: The proof follows the same steps as the ones of Theorem 1, Lemma 1 is applied with

$M_{0}=\left[\begin{array}{cc}\operatorname{He}\left\{\Psi_{11}\right\} & \star \\ \Lambda_{1} & -\gamma I\end{array}\right], \quad M_{1}^{T}=-\left[\begin{array}{c}\varphi_{12} \\ \left(C_{z 2}+D D_{c} C_{2}\right)\end{array}\right] A_{22}^{-1}$,

$M_{2}=\left[\begin{array}{ll}\varphi_{21} & 0\end{array}\right], N_{1}^{T}=-A_{22}\left[\begin{array}{ll}\mathscr{I}^{T} & 0\end{array}\right]$ and $N_{2}=-\varsigma A_{22}^{T}$, we conclude that if (36) holds, then (35) is satisfied and (18) is verified.

Finally, pre- and post-multiplying (37) by $\left[\begin{array}{ll}I & B_{2} D_{c}\end{array}\right]$ and its transpose, one gets (19).

Remark 3 If we consider the initial conditions of the controller are zero, $\xi(0)=(x(0), 0)$ and $\mu(0)=(z(0), 0)$, one has $\bar{J}=\gamma x(0)^{T} Y_{1} x(0)+\gamma \mu_{f}(0)^{T} W_{2}^{-1} \mu_{f}(0), \mu_{f}(0)=(z(0), 0)+$ $G(x(0), 0), G=\tilde{A}_{22}^{-1} \tilde{A}_{21}$. In this case, we can minimize $\bar{J}$ for a given $\gamma$ by the minimization of the trace of $Y_{1}$ and $W_{2}^{-1}$. Considering the structure of the Lyapunov matrix given in (22), one has $\operatorname{Tr}\left(W_{2}^{-1}\right)=\operatorname{Tr}\left(Y_{2}+Y_{2}-X_{2}^{-1}\right)$ where $Y_{2}-X_{2}^{-1}>0$ from (28). Therefore, the minimization of the guaranteed cost is obtained by solving the following optimization problem: $\min \operatorname{Tr}(Q)$ subject to $Q>\operatorname{diag}\left(Y_{1}, Y_{2}\right)$ and (28)-(29) (or (28) and (36)-(37)).

Remark 4 Notice that the controller $u(t)=C_{c} x_{c}(t)+$ $D_{c} C_{1} x(t)+D_{c} C_{2} z(t)$ involves both slow and fast variables. Nevertheless, if we consider that the boundary-layer (fast) system is open-loop stable, i.e., $A_{22}$ Hurwitz, one can design strictly proper controllers by imposing $D_{c}=0$ in Theorem 2 . Doing so, the control signal $u(t)$ does not depend anymore on the fast variable $z(t)$ leading to slow actuation that can be easily implemented in practice.

\section{NUMERICAL EXAMPLE}

Let system (1) given by the nominal singularly perturbed in [1], [4] with the following matrices:

$$
\begin{gathered}
A_{11}=\left[\begin{array}{cc}
-0.195 & -0.676 \\
1.478 & 0
\end{array}\right], A_{12}=\left[\begin{array}{cc}
-0.917 & 0.109 \\
0 & 0
\end{array}\right], \\
A_{21}=\left[\begin{array}{cc}
-0.052 & 0 \\
0.014 & 0
\end{array}\right], A_{22}=\left[\begin{array}{cc}
-0.368 & 0.438 \\
-2.103 & -0.215
\end{array}\right], \\
B_{1}=\left[\begin{array}{c}
-0.023 \\
-16.945
\end{array}\right], B_{2}=\left[\begin{array}{c}
-0.048 \\
-3.811
\end{array}\right], D=\left[\begin{array}{c}
0 \\
10
\end{array}\right], \\
C_{1}=\left[\begin{array}{ll}
0 & 1 \\
0 & 0
\end{array}\right], C_{2}=\left[\begin{array}{cc}
0.921 & -0.161 \\
0 & 1
\end{array}\right],
\end{gathered}
$$

$C_{z 1}=\operatorname{diag}(0.1,0), C_{z 2}=\operatorname{diag}(0,0)$, and the singular perturbation parameter is $\varepsilon=0.0336$ [1]. As in works [1], [4], Theorem 1 with $\varsigma=1$ and $\mathscr{I}=0$ is able to find a solution providing the following gains for the $\varepsilon$-dependent controller 
(5)

$$
\begin{aligned}
& {\left[\begin{array}{l|l}
A_{c 11} & A_{c 12} \\
\hline A_{c 21} & A_{c 22}
\end{array}\right]=\left[\begin{array}{cc|cc}
-0.35 & -0.49 & -1.0 & -1.3 \\
1.3 & -1.9 & -1.4 & -5.7 \\
\hline-0.16 & 0.023 & -0.76 & 0.19 \\
0.17 & 2.4 & 0.1 & -0.53
\end{array}\right],} \\
& {\left[\begin{array}{l}
B_{c 1} \\
\hline B_{c 2}
\end{array}\right]=\left[\begin{array}{cc}
0.16 & -1.3 \\
-1.4 & -5.6 \\
\hline 0.016 & -0.14 \\
2.1 & 0.038
\end{array}\right], D_{c}=\left[\begin{array}{ll}
-0.59 & 0.31
\end{array}\right] 10^{-9} \text {, }}
\end{aligned}
$$

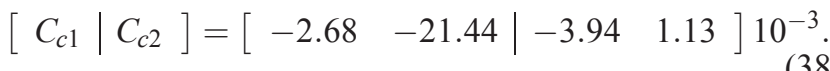

The time-simulations of the closed-loop system for the initial condition $(x(0), z(0))=(1,-1,1,-1)$ and zero initial condition of the controller is presented in Fig. 1 that shows the state trajectories of the plant and the controller. As expected, the fast variables $z$ and $z_{c}$ change abruptly in the initial instant demanding a fast response of the actuators.

Next, we would like to presented some scenarios that cannot be handled by the classical approaches [1], [4]. First, consider the case of designing $\varepsilon$-independent DOF controllers. The following gains for the controller (8) are obtained by Theorem 2 with $\varsigma=10^{-3}$, and $\mathscr{I}=-0.01 I$ :

$$
\begin{aligned}
A_{c 11} & =\left[\begin{array}{cc}
3.13 & 14.34 \\
-1.01 & -4.04
\end{array}\right], B_{c 1}=\left[\begin{array}{cc}
-0.81 & -18.63 \\
-1.44 & -5.71
\end{array}\right], \\
C_{c 1} & =\left[\begin{array}{ll}
-0.58 & -1.69
\end{array}\right], D_{c}=\left[\begin{array}{ll}
-0.063 & 1.063
\end{array}\right] .
\end{aligned}
$$

We can also impose $D_{c}=0$ in order to obtain a control signal $u(t)$ independent of the fast variable as matter of actuator rate constraints, as point out in Remark 4, yielding the following gains for the controller (8) obtained by Theorem 2 with $\varsigma=$ $10^{-9}$ and $\mathscr{I}=0$ :

$$
\begin{gathered}
A_{c}=\left[\begin{array}{cc}
-0.39 & -2.10 \\
1.33 & -1.39
\end{array}\right], B_{c}=\left[\begin{array}{ll}
-1.36 & -3.96 \\
-1.30 & -1.67
\end{array}\right], \\
C_{c}=\left[\begin{array}{ll}
-2.00 & -0.32
\end{array}\right] 10^{-2} .
\end{gathered}
$$

We would like to stress the advantage of the proposed conditions over the existing results in the literature by imposing matrix $A_{22}$ singular and with an unstable eigenvalue. The arbitrary choice is made (eigenvalues 0 and 0.07 )

$$
A_{22}=\left[\begin{array}{ll}
-0.368 & 0.438 \\
-0.368 & 0.438
\end{array}\right]
$$

and the following gains for the controller (5) are obtained with Theorem 1 with $\varsigma=1$ and $\mathscr{I}=0$ :

$$
\begin{aligned}
& {\left[\begin{array}{l|l}
A_{c 11} & A_{c 12} \\
\hline A_{c 21} & A_{c 22}
\end{array}\right]=\left[\begin{array}{cc|cc}
-0.24 & -0.56 & -61.2 & -386.7 \\
1.26 & -3.13 & -780.8 & -4751.9 \\
\hline-0.11 & -1.43 & -48.7 & -283.9 \\
0.07 & 3.36 & -102.8 & -646.9
\end{array}\right]} \\
& {\left[\begin{array}{l}
B_{c 1} \\
\hline B_{c 2}
\end{array}\right]=\left[\begin{array}{cc}
0.11 & -19.01 \\
-1.62 & -81.69 \\
\hline-1.35 & -12.00 \\
3.51 & 1.41
\end{array}\right], D_{c}=\left[\begin{array}{ll}
2.90 & 2.12
\end{array}\right] \cdot 10^{-8} \text {, }}
\end{aligned}
$$

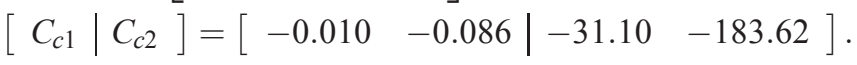

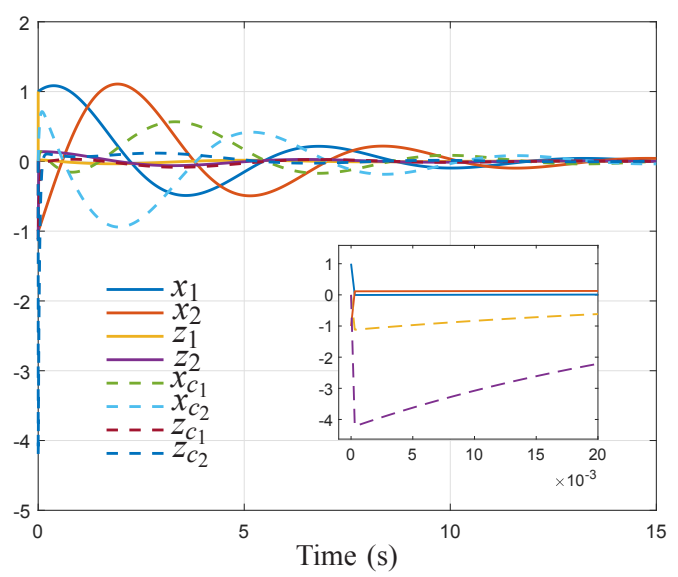

Fig. 1: State trajectories of the plant (solid line) and the controller (dashed line) with gains (38). The figure inset illustrates the trajectories of the fast variables $z$ (solid line) and $z_{c}$ (dashed line) in the initial period.

\section{CONCLUSION}

In this paper, the output feedback guaranteed cost control problem for linear singularly perturbed systems is explored. We propose a time-scale decomposition of the closed-loop system for the design of full and reduced-order controllers. The design, implemented by $\varepsilon$-independent sufficient LMI conditions, do not require the fast dynamic matrix to be nonsingular or stable. The controller implementation does not require the knowledge on the singular perturbation parameter and the use of fast actuators to stabilize the fast dynamics. Numerical simulation emphasizes the effectiveness of our results.

\section{REFERENCES}

[1] P. Kokotovic, H. Khalil, and J. O'Reilly, Singular perturbation methods in control: Analysis and design. Philadelphia: SIAM, 1999.

[2] G. Garcia, J. Daafouz, and J. Bernussou, "The infinite time near optimal decentralized regulator problem for singularly perturbed systems: a convex optimization approach," Automatica, vol. 38, no. 8, pp. 1397 $-1406,2002$.

[3] H. Khalil, "Output feedback control of linear two-time-scale systems," IEEE Transactions on Automatic Control, vol. 32, no. 9, pp. 784-792, 1987.

[4] J. Daafouz, G. Garcia, and J. Bernussou, " $H_{2}$ guaranteed cost control by dynamic output feedback for uncertain singularly perturbed systems," IFAC Proceedings Volumes, vol. 32, no. 2, pp. 3520 - 3525, 1999, 14th IFAC World Congress 1999, Beijing, Chia, 5-9 July.

[5] P. D. Christofides, "Robust output feedback control of nonlinear singularly perturbed systems," Automatica, vol. 36 , no. 1, pp. 45$52,2000$.

[6] J. Xu, C. Cai, Y. Zou, and C. C. Lim, "Mixed output feedback of the finite frequency $\mathrm{H}_{\infty}$ control for singularly perturbed system," International Journal of Systems Science, vol. 46, no. 13, pp. 23512366, 2015.

[7] M. C. de Oliveira and R. E. Skelton, "Stability tests for constrained linear systems," in Perspectives in Robust Control, ser. Lecture Notes in Control and Information Science, S. O. Reza Moheimani, Ed. New York, NY: Springer-Verlag, 2001, vol. 268, pp. 241-257.

[8] G. S. Deaecto, J. Daafouz, and J. C. Geromel, " $H_{2}$ and $H_{\infty}$ performance optimization of singularly perturbed switched systems," SIAM Journal on Control and Optimization, vol. 50, no. 3, pp. 1597-1615, 2012.

[9] C. Scherer, P. Gahinet, and M. Chilali, "Multiobjective outputfeedback control via LMI optimization," IEEE Transactions on Automatic Control, vol. 42, no. 7, pp. 896-911, July 1997. 